Tohoku J. Exp. Med., 2003, 201, 171-179

\title{
Oral Health of Patients with Parkinson's Disease: Factors Related to Their Better Dental Status
}

\author{
Shingo Fukayo, Koichi Nonaka, ${ }^{1}$ Teruo Shimizu² and Eiji Yano \\ Department of Hygiene and Public Health, Teikyo University School of \\ Medicine, Tokyo 173-8605, \\ ${ }^{1}$ Department of Human Development, Faculty of Human Sciences, Wako \\ University, Tokyo 195-8585, and \\ ${ }^{2}$ Department of Neurology, Teikyo University School of Medicine, Tokyo 173- \\ 8605
}

Fukayo, S., Nonaka, K., Shimizu, T. and Yano, E. Oral Health of Patients with Parkinson's Disease: Factors Related to Their Better Dental Status. Tohoku J. Exp. Med., 2003, 201 (3), 171-179_Contrary to intuitive preconceptions, patients with Parkinson's disease (PD) have been reported to have less carious teeth. The present study was undertaken to seek responsible factors for this unexpected finding. The PD patients consisted of 31 consecutive university hospital outpatients who were 60 years old or over, and the controls of 104 comparable outpatients at a dental clinic. They were inspected for their dental status, and interviewed on their toothbrushing habits and dietary preferences. Their unstimulated saliva samples were collected and their flow volume and $\mathrm{pH}$ were measured. The total numbers of carious teeth (DMFT: Decayed, Missing, and Filled Teeth) and other related variables were compared between the two groups by stratification. In total, the DMFT for the PD patients was significantly fewer than for the control. The salivary flow and $\mathrm{pH}$ were correlated to the DMFT, but the difference between the two groups was not significant. Frequency of toothbrushing was higher among the PD patients. The lower DMFT among the patients became insignificant when salivary $\mathrm{pH}$ was 6 or less, toothbrushing was 2 times a day or less, or the response was positive to the question on the habit of eating snacks between regular meals. In summary, the oral health of PD outpatients with mild symptoms was better than the controls. However, in cases with poor oral hygiene status, the PD patient's oral health was not different from the control. This suggests that they are not invariably protected from caries-associated factors. The generic property of PD may not fully explain the apparent difference in DMFT._— Parkinson's disease; dental caries; DMF index; saliva; toothbrushing

(C) 2003 Tohoku University Medical Press

Received June 2, 2003; revision accepted for publication September 29, 2003.

Address for reprints: Prof. Eiji Yano, Department of Hygiene and Public Health, Teikyo University

School of Medicine, 2-11-1 Kaga, Itabashi-ku, Tokyo 173-8605, Japan.

e-mail: eyano@med.teikyo-u.ac.jp 
To maintain the quality of life (QOL) of the elderly, it is important to develop and retain good oral health (Locker 1992; Kressin et al. 1996; Leao and Sheiham 1996). The advent of physical impairment with aging can lead to inadequate oral hygiene, a consequent reduction in the number of functioning teeth, and thus further suffering from a deteriorating QOL. One of the most common elderly neurological disorders is Parkinson's disease (PD). Subjects may be expected to experience increased difficulties in oral health practices due to its resting tremor, bradykinesia, akinesia, restricted mobility and postural instability, which together with their reduced salivary flow, may cause aggravation of their oral health problems (Jolly et al. 1989; Clifford and Finnerty 1995). Swallowing dysfunction in PD patients (Edwards et al. 1992, 1993 and 1994) could be also related to oral health through associated changes in salivary retention. The leading cause of death among PD patients was reported to be pneumonia (Nakashima et al. 1997), which could have been aggravated by having aspired oral contents remaining due to poor oral health. Recognition of these factors, in the dental management of individuals with $\mathrm{PD}$, is a multifaceted challenge involving areas of preventive, restorative, and prosthetic dentisty (Jolly et al. 1989).

Our previous survey of the oral health status of $\mathrm{PD}$ patients revealed better than expected oral health, namely, a smaller number of carious teeth and a larger number of remaining teeth (Fukayo 2002). However, this study was based on the comparison of referent population data from the National Dental Disease Survey in 1993 in Japan (Health Policy Bureau, Ministry of Health and Welfare, Japan 1995). Consequently it was not possible to determine as to whether their relatively good dental status was due to better dietary or toothbrushing habits, or to some other physical characteristics specific to the PD patients. Thus in the present study, we have endeavoured to identify the associated potential factors responsible for the differential dental status of the patients by using a control sample group of consecutive outpatients attending a dental clinic.

\section{MATERIAL AND METHODS}

The patients with PD consisted of 31 (17 males and 14 females) consecutive outpatients who were 60 years old or over and who visited a university hospital, and the control of 104 (61 males and 43 females) consecutive outpatients aged 60 years old or over at a dental clinic. All the PD patients were of a mild type in stages 13 of the Hoehn and Yahr score (1967). The purpose of the study was explained to them before starting the examination, and the informed consent for participation was given by all of the subjects.

All the subjects were inspected by one of the authors (S.F.) for their dental caries status. The number of carious teeth, designated DMFT (Decayed, Missing and Filled Teeth), was used for estimating the oral status of the subjects. The teeth considered for calculating DMFT were the central and lateral incisors, the canines, the first and second premolars, and the first and second molars, which summates to 28 teeth per subject. When tooth-specific DMFT was calculated, the teeth were grouped into four subgroups; namely, anterior (incisors and canines), posterior (premolars and molars), maxillary and mandibular teeth.

Their unstimulated saliva samples were collected during the dental inspection (Edger and O'Mullane 1996). The subjects, for both groups, were instructed to avoid eating or drinking for thirty minutes before collection, and their unstimulated five-minutes saliva samples were collected in paper cups. The volume and $\mathrm{pH}$ of the saliva were measured immediately after the collection using a pH meter (HI 8114, Hanna Co. Ltd., Tokyo).

The subjects were also interviewed, before the dental inspection, on their toothbrushing habits and dietary preferences using a set of prepared questions. They were asked to 
answer Yes/No for toothbrushings: (i) immediately after wake-up in the morning, (ii) after breakfast, (iii) after lunch, (iv) after dinner, and (v) before bed, respectively, and the number of "Yes" answers per individual was used as the frequency of toothbrushing per day. To obtain information on their caries-related dietary habits, their preferences for (i) sweet, (ii) soft food, (iii) hard food were solicited, and the inter-meal habit, that is their custom of eating snacks between regular meals or after dinner, were also noted. The dichotomized Yes/No answers to these questions were used for the analysis.

The DMFT, the flow volume and $\mathrm{pH}$ of saliva, eating preferences and the frequency of toothbrushing per day were compared between the case and the control groups, stratified by sex- and two age-groups (60-69 and 70+ years groups). Wilcoxon's rank sum tests were used for continuous variables because the distribution of the DMFT was not normal, and Fisher's exact test for discrete ones. Relationships between the DMFT and continuous variables are illustrated in the scattergram, and the Spearman's correlation coefficients were calculated for each group because the correlation was not linear. Effects of caries-related discrete variables, including dichotomized continuous variables, on the difference between the two groups were examined by stratification. The significance levels were shown as exact $p$ values, and the results were regarded significant when the $p$-value was less than 0.05. Analyses were performed using statistical packages: SAS (SAS Institute Inc. 1998) and the Statistical Packages for the Biosciences (SPBS) (Murata and Yano 2002).

\section{RESULTS}

DMFT of the PD patients and the controls according to the sex and age group are shown in Table 1. In general, the DMFT was significantly less among the PD patients. When subdivided according to sex and age groups, the difference was basically consistent, with an exception of the subgroup of male 70 years old or over.

Table 2 shows differences in salivary flow and salivary $\mathrm{pH}$ between the two groups. They were of no statistical significance with an exception of a lower salivary flow for the controls aged 70 years or over. Table 3 shows the mean frequency of toothbrushing per day for

TABle 1. DMFT among PD patients and controls

\begin{tabular}{|c|c|c|c|c|c|}
\hline \multirow{2}{*}{$\begin{array}{l}\text { Sex } \\
\text { Age(yrs) }\end{array}$} & \multicolumn{2}{|c|}{ PD patients } & \multicolumn{2}{|c|}{ Controls } & \multirow{2}{*}{$p$-value $^{1}$} \\
\hline & $n$ & mean \pm s.e. & $n$ & mean \pm s.E. & \\
\hline \multicolumn{6}{|l|}{ Total } \\
\hline Total & 31 & $19.3 \pm 1.5$ & 104 & $25.8 \pm 0.3$ & 0.000 \\
\hline 60-69 & 12 & $14.2 \pm 2.1$ & 76 & $25.3 \pm 0.4$ & 0.000 \\
\hline $70+$ & 19 & $22.5 \pm 1.6$ & 28 & $27.0 \pm 0.5$ & 0.017 \\
\hline \multicolumn{6}{|l|}{ Male } \\
\hline Total & 17 & $20.3 \pm 2.0$ & 61 & $25.2 \pm 0.5$ & 0.103 \\
\hline $60-69$ & 6 & $11.5 \pm 2.7$ & 46 & $24.9 \pm 0.6$ & 0.000 \\
\hline $70+$ & 11 & $25.1 \pm 1.2$ & 15 & $26.2 \pm 0.8$ & 0.495 \\
\hline \multicolumn{6}{|l|}{ Female } \\
\hline Total & 14 & $18.0 \pm 2.2$ & 43 & $26.6 \pm 0.4$ & 0.001 \\
\hline $60-69$ & 6 & $16.8 \pm 3.1$ & 30 & $26.0 \pm 0.5$ & 0.006 \\
\hline $70+$ & 8 & $18.9 \pm 3.2$ & 13 & $27.9 \pm 0.1$ & 0.012 \\
\hline
\end{tabular}

${ }^{1}$ Wilcoxon's rank sum test. 
TABle 2. Salivary flow and salivary $p H$ among $P D$ patients and controls

\begin{tabular}{|c|c|c|c|c|c|c|c|c|}
\hline \multirow{3}{*}{$\begin{array}{l}\text { Sex } \\
\text { Age(yrs) }\end{array}$} & \multicolumn{5}{|c|}{ Salivary flow (ml/5 min) } & \multicolumn{3}{|c|}{ Salivary $\mathrm{pH}$} \\
\hline & \multicolumn{2}{|c|}{ PD patients } & \multicolumn{2}{|c|}{ Controls } & \multirow{2}{*}{$p$-value ${ }^{1}$} & \multirow{2}{*}{$\frac{\text { PD patients }}{\text { mean } \pm \text { s.E. }}$} & \multirow{2}{*}{$\begin{array}{c}\text { Controls } \\
\text { mean } \pm \text { s.E. }\end{array}$} & \multirow{2}{*}{$p$-value ${ }^{1}$} \\
\hline & $n$ & mean \pm s.E. & $n$ & mean \pm s.E. & & & & \\
\hline Total & 31 & $2.2 \pm 1.3$ & 104 & $2.0 \pm 0.1$ & 0.708 & $6.5 \pm 0.5$ & $6.7 \pm 0.1$ & 0.075 \\
\hline $60-69$ & 12 & $2.3 \pm 1.2$ & 76 & $2.3 \pm 0.1$ & 0.947 & $6.6 \pm 1.5$ & $6.7 \pm 0.1$ & 0.515 \\
\hline $70+$ & 19 & $2.2 \pm 1.3$ & 28 & $1.3 \pm 0.1$ & 0.037 & $6.4 \pm 0.6$ & $6.6 \pm 0.1$ & 0.308 \\
\hline Male & 17 & $2.3 \pm 0.3$ & 61 & $2.2 \pm 0.2$ & 0.904 & $6.4 \pm 0.1$ & $6.7 \pm 0.1$ & 0.117 \\
\hline $60-69$ & 6 & $2.3 \pm 1.5$ & 46 & $2.5 \pm 0.2$ & 0.731 & $6.6 \pm 0.4$ & $6.7 \pm 0.1$ & 0.616 \\
\hline $70+$ & 11 & $2.3 \pm 1.4$ & 15 & $1.4 \pm 0.2$ & 0.067 & $6.3 \pm 0.6$ & $6.6 \pm 0.2$ & 0.275 \\
\hline Female & 14 & $2.0 \pm 0.3$ & 43 & $1.7 \pm 0.1$ & 0.602 & $6.5 \pm 0.2$ & $6.7 \pm 0.1$ & 0.344 \\
\hline $60-69$ & 6 & $2.2 \pm 1.0$ & 30 & $1.9 \pm 0.2$ & 0.609 & $6.6 \pm 0.7$ & $6.8 \pm 0.1$ & 0.656 \\
\hline $70+$ & 8 & $2.0 \pm 1.4$ & 13 & $1.3 \pm 0.1$ & 0.358 & $6.5 \pm 0.5$ & $6.6 \pm 0.2$ & 0.856 \\
\hline
\end{tabular}

${ }^{1}$ Wilcoxon's rank sum test.

TABLE 3. Tooth-brushing habits among PD patients and controls

\begin{tabular}{|c|c|c|c|c|c|c|}
\hline \multirow{3}{*}{$\begin{array}{l}\text { Sex } \\
\text { Age(yrs) }\end{array}$} & \multicolumn{3}{|c|}{ Number of brushing per day } & \multicolumn{3}{|c|}{ Brushing 3 times or more/day } \\
\hline & \multirow{2}{*}{$\begin{array}{c}\text { PD patients } \\
\text { mean } \pm \text { s.e. } \\
\text { (times/day) }\end{array}$} & \multirow{2}{*}{$\begin{array}{c}\text { Controls } \\
\text { mean } \pm \text { s.E. } \\
\text { (times/day) }\end{array}$} & \multirow[b]{2}{*}{$p$-value ${ }^{1}$} & \multirow{2}{*}{$\frac{\mathrm{PD} \text { patients }}{\%}$} & \multirow{2}{*}{$\begin{array}{c}\text { Controls } \\
\%\end{array}$} & \multirow{2}{*}{$p$-value ${ }^{2}$} \\
\hline & & & & & & \\
\hline Total & $2.8 \pm 0.2$ & $2.1 \pm 0.1$ & 0.000 & 68 & 25 & 0.000 \\
\hline $60-69$ & $3.3 \pm 0.3$ & $2.2 \pm 0.1$ & 0.000 & 83 & 30 & 0.001 \\
\hline $70+$ & $2.6 \pm 0.3$ & $1.9 \pm 0.1$ & 0.020 & 58 & 11 & 0.001 \\
\hline Male & $2.5 \pm 0.3$ & $1.9 \pm 0.1$ & 0.015 & 59 & 20 & 0.004 \\
\hline 60-69 & $3.3 \pm 0.2$ & $2.0 \pm 0.1$ & 0.001 & 100 & 24 & 0.001 \\
\hline $70+$ & $2.1 \pm 0.4$ & $1.7 \pm 0.2$ & 0.356 & 36 & 7 & 0.128 \\
\hline Female & $3.2 \pm 0.3$ & $2.3 \pm 0.1$ & 0.004 & 79 & 33 & 0.004 \\
\hline $60-69$ & $3.2 \pm 0.5$ & $2.5 \pm 0.2$ & 0.140 & 67 & 40 & 0.374 \\
\hline $70+$ & $3.3 \pm 0.4$ & $2.0 \pm 0.2$ & 0.013 & 88 & 15 & 0.002 \\
\hline
\end{tabular}

${ }^{1}$ Wilcoxon's rank sum test.

${ }^{2}$ Fisher's exact test.

Table 4. Dietary preferences and intermeal

\begin{tabular}{|c|c|c|c|c|c|c|c|c|c|c|}
\hline \multirow{3}{*}{$\begin{array}{l}\text { Sex } \\
\text { Age(yrs) }\end{array}$} & \multicolumn{5}{|c|}{ Preference for sweet } & \multicolumn{5}{|c|}{ Preference for soft stuff } \\
\hline & \multicolumn{2}{|c|}{ PD patients } & \multicolumn{2}{|c|}{ Controls } & \multirow{2}{*}{$p$-value ${ }^{1}$} & \multicolumn{2}{|c|}{ PD patients } & \multicolumn{2}{|c|}{ Controls } & \multirow{2}{*}{$p$-value ${ }^{1}$} \\
\hline & Yes/Total & $(\%)$ & Yes/Total & $(\%)$ & & Yes & $(\%)$ & Yes & $(\%)$ & \\
\hline Total & $19 / 31$ & 61 & $65 / 104$ & 63 & 1.000 & 22 & 71 & 45 & 43 & 0.008 \\
\hline $60-69$ & $9 / 12$ & 75 & $47 / 76$ & 62 & 0.524 & 8 & 67 & 29 & 38 & 0.113 \\
\hline $70+$ & $10 / 19$ & 53 & $18 / 28$ & 64 & 0.547 & 14 & 74 & 16 & 57 & 0.356 \\
\hline Male & $11 / 17$ & 65 & $34 / 61$ & 56 & 0.587 & 13 & 76 & 24 & 39 & 0.012 \\
\hline $60-69$ & $5 / 6$ & 83 & $26 / 46$ & 57 & 0.382 & 4 & 67 & 18 & 39 & 0.382 \\
\hline $70+$ & $6 / 11$ & 55 & $8 / 15$ & 53 & 1.000 & 9 & 82 & 6 & 40 & 0.051 \\
\hline Female & $8 / 14$ & 57 & $31 / 43$ & 72 & 0.333 & 9 & 64 & 21 & 49 & 0.369 \\
\hline $60-69$ & $4 / 6$ & 67 & $21 / 30$ & 70 & 1.000 & 4 & 67 & 11 & 37 & 0.210 \\
\hline $70+$ & $4 / 8$ & 50 & $10 / 13$ & 77 & 0.346 & 5 & 63 & 10 & 77 & 0.631 \\
\hline
\end{tabular}

${ }^{1}$ Fisher's exact test. 
the two groups. The PD patients brushed their teeth more frequently ( $2.8 \pm 0.2$ times per day) than the control $(2.1 \pm 0.1)$ in all subgroups, and the percentage of those who brushed their teeth 3 times a day or more was significantly higher among the PD patients.

Table 4 indicates dietary preferences and inter-meal habit for the two groups. The PD patients preferred soft food significantly more than the controls (71\% vs. $43 \%$ ), and had less inter-meal habit (26\% vs. 35\%) though statistical significance was reached only for the female subgroup (7\% vs. $42 \%$ ).

Fig. 1 shows the scattergram between the individual DMFT value and four continuous variables: (a) age, (b) salivary $\mathrm{pH}$, (c) salivary flow, and (d) toothbrushing frequency. DMFT increased according to age for both groups, but the correlation coefficient was larger for the PD patients. DMFT decreased according to the volume of salivary flow and to the salivary $\mathrm{pH}$ value for both groups. Salivary flow and salivary $\mathrm{pH}$ were strongly correlated with each other, and the correlation was stronger for the PD patients (Spearman correlation coefficient, $r=+0.69$ ). As to the frequency of toothbrushing, DMFT showed a negative correlation for the PD patients $(r=-0.47)$, but no correlation for the controls $(r=+0.01)$.

Fig. 2 shows the difference in DMFT between the two groups when examined in several substrata of the related factors. Significant differences were not observed in some strata: namely those with salivary $\mathrm{pH}$ being 6 or less, those with the frequency of toothbrushing up to 2 times a day, and those who had inter-meal habit. For such factors as salivary flow, dietary preferences for sweet, soft food, or hard food, the difference between the two groups remained significant both in the subcategories. These tendencies remained observed for each group of teeth.

\section{DISCUSSION}

In reviews on PD patients (Jolly et al. 1989; Johnston et al. 1995), dental complications have been suggested to arise from the inability of the patients to accomplish routine oral hygiene, and from dysfunctional changes in salivary flow and swallowing which would manifest as dysphagia or drooling of saliva. Medication for neurodegenerative diseases can produce xerostomia, which would reduce the oral salivary function to prevent caries (Kieser et al. 1999). Among the present sample of PD patients, those who were being administered anti-cholinergic drug at the time of investigation tended to show less, though not significantly, salivary flow. These factors may affect the cariogenic environments of the PD patients, and the cumulative effects

habit among PD patients and controls

\begin{tabular}{|c|c|c|c|c|c|c|c|c|c|}
\hline \multicolumn{5}{|c|}{ Preference for hard stuff } & \multicolumn{5}{|c|}{ Inter-meal habit } \\
\hline \multicolumn{2}{|c|}{ PD patients } & \multicolumn{2}{|c|}{ Controls } & \multirow{2}{*}{$p$-value ${ }^{1}$} & \multicolumn{2}{|c|}{ PD patients } & \multicolumn{2}{|c|}{ Controls } & \multirow{2}{*}{$p$-value $^{1}$} \\
\hline Yes & $(\%)$ & Yes & $(\%)$ & & Yes & $(\%)$ & Yes & $(\%)$ & \\
\hline 14 & 45 & 57 & 55 & 0.414 & 8 & 26 & 36 & 35 & 0.392 \\
\hline 6 & 50 & 44 & 58 & 0.756 & 0 & 0 & 24 & 32 & 0.031 \\
\hline 8 & 42 & 13 & 46 & 1.000 & 8 & 42 & 12 & 43 & 1.000 \\
\hline 6 & 35 & 34 & 56 & 0.174 & 7 & 41 & 18 & 30 & 0.389 \\
\hline 2 & 33 & 26 & 57 & 0.397 & 0 & 0 & 12 & 26 & 0.316 \\
\hline 4 & 36 & 8 & 53 & 0.453 & 7 & 64 & 6 & 40 & 0.428 \\
\hline 8 & 57 & 23 & 53 & 1.000 & 1 & 7 & 18 & 42 & 0.022 \\
\hline 4 & 67 & 18 & 60 & 1.000 & 0 & 0 & 12 & 40 & 0.079 \\
\hline 4 & 50 & 5 & 38 & 0.673 & 1 & 13 & 6 & 46 & 0.174 \\
\hline
\end{tabular}



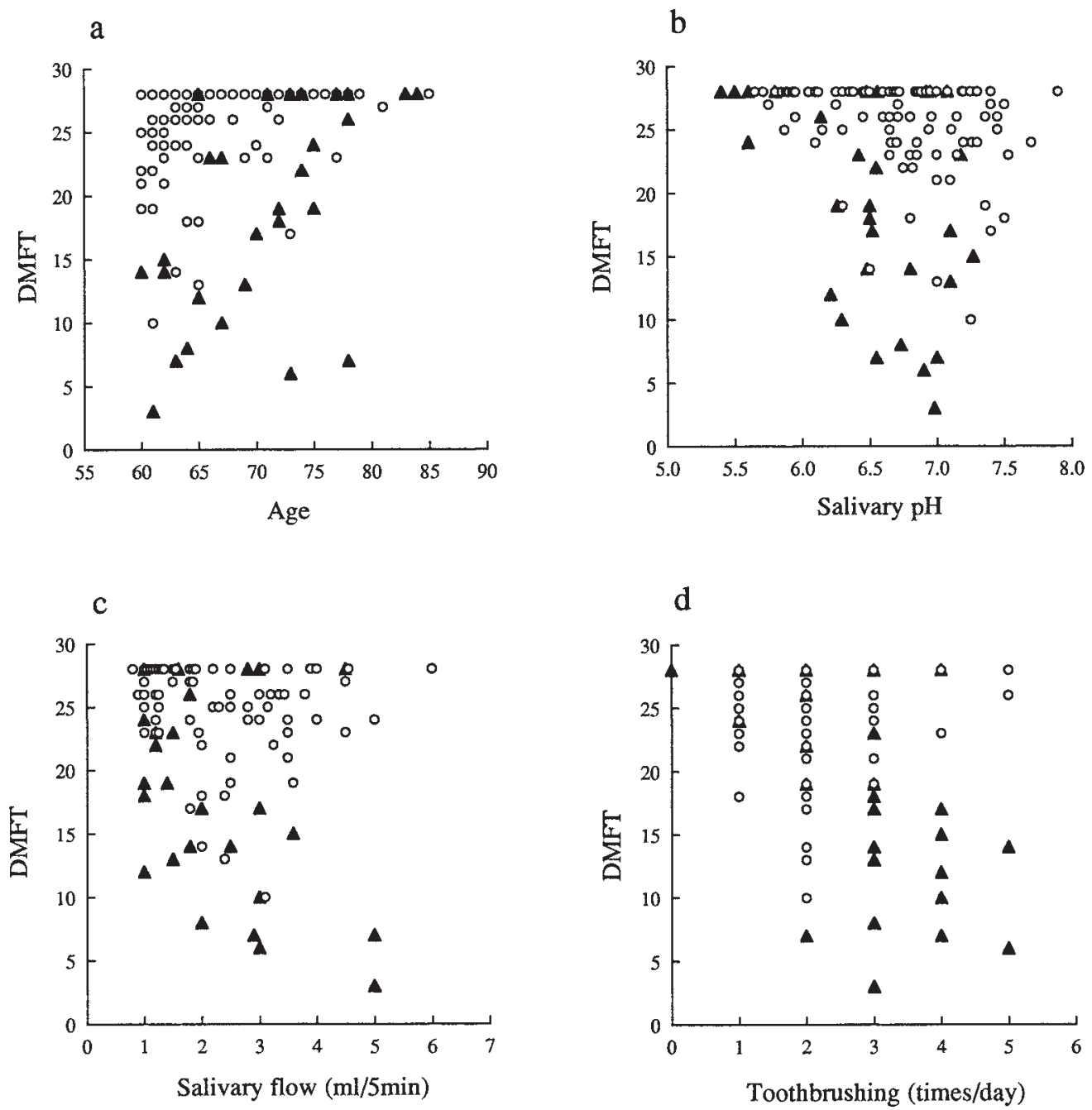

Fig. 1. Relationship between DMFT and (a) age, (b) salivary pH, (c) salivary flow, and (d) toothbrushing frequency. Solid triangles $(\mathbf{\Lambda})$ indicate $31 \mathrm{PD}$ patients and open circles $(\bigcirc)$ 104 controls. Spearman's correlation coefficients $(r)$ were separately calculated for the PD patients and for the control. (a) $\boldsymbol{\Delta}, r=+0.54(p=0.002), \bigcirc, r=+0.38(p=0.001)$, (b) $\boldsymbol{\Delta}, r=-0.39$ ( $p=0.028), \bigcirc, r=-0.35(p=0.000),(c) \mathbf{\Delta}, r=-0.39(p=0.032), \bigcirc, r=-0.45$ $(p=0.000),(\mathrm{d}) \boldsymbol{\Delta}, r=-0.47(p=0.008), \bigcirc, r=+0.01(p=0.903)$.

would be observed as the larger number of caries teeth among the patients. Our previous report (Fukayo 2002) on the patients with mild PD symptoms however suggested, somewhat surprisingly, a better oral status, although the adequacy of the referent population cast some doubt on the validity of the results.

Hence the validity of the present selection of outpatients at a dental clinic as a suitable control group must be first assessed. While the PD patients may receive special care because of their disease, the outpatients at a dental clinic may be more conscious of their oral health than the general population which may include those who never visit dental clinics. Although the data on caries-related habits for the general elderly population was not available, the weighted mean DMFT for the general population of the corresponding age groups, obtained by the National Dental Diseases Survey in Japan in 1993 and 1999 (Health Policy Bureau, Ministry of Health and Welfare, Japan 1995, 2001), was 


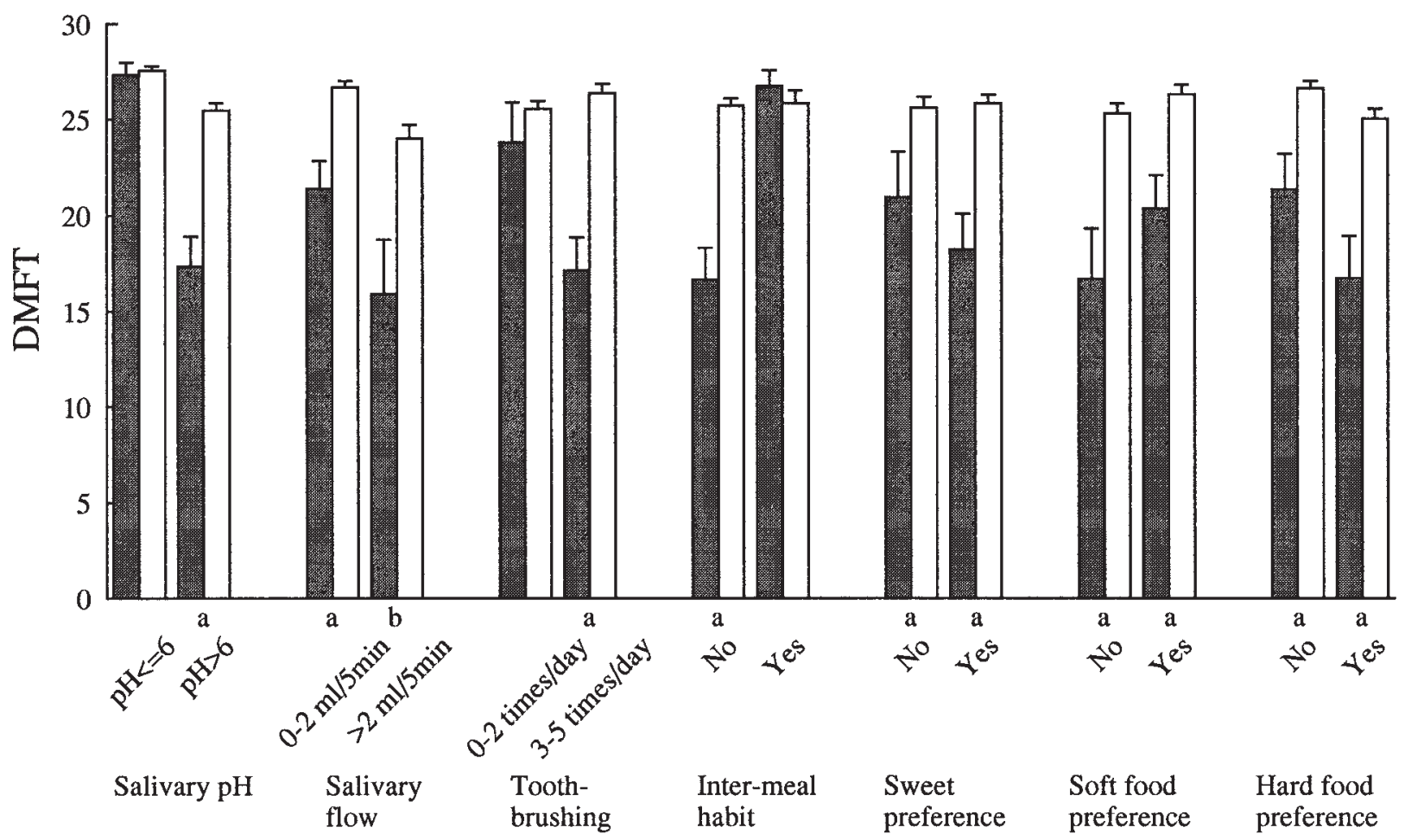

Fig. 2. DMFT difference between the PD patients and the control according to dichotomized several caries-related factors. Solid bars indicate mean DMFT for the PD patients and open bars for the control. Each notch on the bar denotes one standard error of the mean. The asterisks indicate the results of Wilcoxon's rank sum test between the two groups. ${ }^{\mathrm{a}} p<0.01, \quad{ }^{\mathrm{b}} p<0.10$.

24.3 in 1993 and 22.9 in $1999 . \quad$ These were lower than that of the present control sample, 25.8, and may suggest that the present sample was biased to those who needed to have access to a dental clinic, but was still higher than that of the PD patients, 19.3.

The basic finding in our previous study (Fukayo 2002) that PD patients had a lower DMFT was confirmed in this study. This is consistent with a report from Sweden (Persson et al. 1992) that PD patients with mild symptoms had significantly less caries teeth than control. The medications for PD could result in xerostomia and consequent root caries and recurrent decay (Kieser et al. 1999), and cases of high caries activity due to drug therapy have been reported among PD patients (Winer and Bahn 1967). However, the patients of the present study did not show lower salivary flow rates than the controls (Table 2), despite the facts that all of them were being administered levodopa which can reduce salivary secretion (Kieser et al. 1999) and that those who were being administered anti-cholinergic drug tended to show a lower salivary flow. The PD patients, evaluated in this study, were being administered levodopa, but did not show disadvantages in terms of salivary secretion.

When compared in sex- and age-specific subgroups, the difference in DMFT between the PD patients and the control was basically consistent, with the exception of the male $70+$ years old group. Factors likely to affect the prevalence of caries were compared between the PD patients and the controls (Tables 2, 3 and 4). The patients were not different from the controls as to salivary flow and salivary $\mathrm{pH}$, while they kept better routine oral hygiene with a higher frequency of toothbrushing per day, and tended to have less inter-meal habit. 
Although these results indicate that the patients as a group kept better habits for caries prevention, they were also subject to the effects of adverse caries-promoting environments and habits: they had a larger DMFT when their age advanced, salivary $\mathrm{pH}$ was low, salivary flow was low, or their toothbrushing frequency was low (Fig. 1). The exceptional patient group of the male aged $70+$ years disproportionally exhibited these caries-promoting factors. It has been reported (Kennedy et al. 1994) that PD patients had an increased craving for sweets, and S. mutans was found more frequently in plaque samples of the patients. The preference for sweets may not be a consistent characteristic of the PD patients.

Analysis of the effects of dichotomized related factors on the differences in DMFT between the patients and the control revealed the following facts. The differences between the groups disappeared when salivary $\mathrm{pH}$, toothbrushing frequency, or the inter-meal habit was in the caries-associated category. Thus, the these three factors seemed to have confounded the beneficial effects of being a PD patient. However, the difference still existed when the other factors, such as salivary flow, preferences for sweet, soft food, or hard food, fell in a caries-associated category (Fig. 2). These may suggest two things. First, the patients are similarly at risk of caries incidence when salivary $\mathrm{pH}$ is lower than 6 , toothbrushing frequency is 2 times or less per day, or they have an inter-meal habit. Second, they still have a tendency to have less caries than the control if they keep caries-preventing habits or preferences. The reason why the latter difference persists is still to be investigated, together with reassessment of the appropriateness of selecting dental clinic patients as a control. Another factor we can suppose here is that the present measurement of salivary flow does not directly reflect the amount of saliva actually retained in oral cavity. Hypersialorrhea has been reported in PD patients, but their complaints are not due to the overproduction of saliva, but to their difficulties in swallowing (Bagheri et al. 1999). It may be possible that the salivary function for preventing the activities of cariogenic microflora is augmented among the patients because of their dysphagia symptoms, which can occur at an early stage of the disease regardless of the severity of PD (Edwards et al. 1994; Nilsson et al. 1996; Bassotti et al. 1998).

Although we do not have sufficient-sample size to perform any viable multivariate analysis to adjust for probable mutually confounding factors, it is desirable to seek for specific ways to further reduce the caries incidence among the patients. For example, are the effects of toothbrushing and the inter-meal habits interrelated? Our preliminary analyses indicated that the toothbrushing frequency was significantly lower among those who had an inter-meal habit, both for the PD patients and the control. Thus because the factors of toothbrushing and intermeal habit may not be independent, we cannot specify at present which factor should be considered a priority intervention.

Persson et al. (1992) reported decreases in the salivary flow with advancing severity of PD. Since the present study deals only with patients with mild symptoms, it should be noted that the results shown here may not be applicable to advanced PD patients.

In conclusion, the oral health among PD outpatients with mild symptoms is generally better than comparable general population. When some of the caries-associated environments were particularly poor, however, the oral health status of the PD patients did not differ from that of the controls. This suggests that PD patients are not invariably protected from caries-associated factors, and the generic property of PD may not fully explain the apparent difference in DMFT.

\section{References}

Bagheri, H., Damase-Michel, C., Lapeyre-Mestre, M., Cismondo, S., O'Connell, D. \& Senard, J.M. 
(1999) A study of salivary secretion in Parkinson's disease. Clin. Neuropharmacol., 22, 213-215.

Bassotti, G., Germani, U., Pagliaricci, S., Plesa, A., Giulietti, O. \& Mannarino, E. (1998) Esophageal manometric abnormalities in Parkinson's disease. Dysphagia, 13, 28-31.

Clifford, T. \& Finnerty, J. (1995) The dental awareness and needs of a Parkinson's disease population. Gerodontology, 12, 99-103.

Edgar, W.M. \& O’Mullane, D.M. (1996) Saliva and Oral Health, 2nd ed., London, British Dental Association, London.

Edwards, L.L., Quigley, E.M. \& Pfeiffer, R.F. (1992) Gastrointestinal dysfunction in Parkinson's disease: frequency and pathophysiology. Neurology, 42, 726-732.

Edwards, L.L., Pfeiffer, R.F., Quigley, E.M., Hofman, R. \& Balluff, M. (1993) Gastrointestinal symptoms in Parkinson's disease. Mov. Disord., 6, 151-156.

Edwards, L.L., Quigley, E.M., Harned, R.K., Hofman, R. \& Pfeiffer, R.F. (1994) Characterization of swallowing and defecation in Parkinson's disease. Am. J. Gastroenterol., 89, 15-25.

Fukayo, S. (2002) Dental status in outpatients with Parkinson's disease. Jpn. J. Hyg., 57, 585-590. (in Japanese)

Health Policy Bureau, Ministry of Health and Welfare, Japan (1995). Report on the Survey of Dental Diseases (1993). Koku Hoken Kyokai, Tokyo.

Health Policy Bureau, Ministry of Health and Welfare, Japan (2001). Report on the Survey of Dental Diseases (1999). Koku Hoken Kyokai, Tokyo.

Hoehn, M.M. \& Yahr, M.D. (1967) Parkinsonism: onset, progression and mortality. Neurology, 17, 427-442.

Johnston, B.T., Li, Q., Castell, J.A. \& Castell, D.O. (1995) Swallowing and esophageal function in Parkinson's disease. Am. J. Gastroenterol., 90, 1741-1746.

Jolly, D.E., Paulson, R.B., Paulson, G.W. \& Pike, J.A. (1989) Parkinson's disease: a review and rec- ommendations for dental management. Spec. Care Dentist, 9, 74-78.

Kennedy, M.A., Rosen, S., Paulson, G.W., Jolly, D.E. \& Beck, F.M. (1994) Relationship of oral microflora with oral health status in Parkinson's disease. Spec. Care Dentist, 14, 164-168.

Kieser, J., Jones, G., Borlase, G. \& MacFadyen, E. (1999) Dental treatment of patients with neurodegenerative disease. $\quad N$. Z. Dent. J., 95, 130-134.

Kressin, N., Spiro, A., III., Bosse, R., Garcia, R. \& Kazis, L. (1996) Assessing oral healthrelated quality of life: findings from the normative aging study. Med. Care., 34, 416-427.

Leao, A. \& Sheiham, A. (1996) The development of a socio-dental measure of dental impacts on daily living. Community Dent. Health., 13, 22-26.

Locker, D. (1992) The burden of oral disorders in a population of older adults. Community Dent. Health, 9, 109-124.

Murata, K. \& Yano, E. (2002) Medical Statistics for Evidence Based Medicine with SPBS User's Guide. Nankodo Co., Tokyo.

Nakashima, K., Maeda, M., Tabata, M., Adachi, Y., Kusumi, M. \& Ohshiro, H. (1997) Prognosis of Parkinson's disease in Japan. Tottori University Parkinson's Disease Epidemiology (TUPDE) Study Group. Eur. Neurol., 38, Suppl. 2, 60-63.

Nilsson, H., Ekberg, O., Olsson, R. \& Hindfelt, B. (1996) Quantitative assessment of oral and pharyngeal function in Parkinson's disease. Dysphagia, 11, 144-150.

Persson, M., Osterberg, T., Granerus, A.K. \& Karlsson, S. (1992) Influence of Parkinson's disease on oral health. Acta Odontol. Scand., 50, 37-42.

SAS Institute Inc. (1988) SAS/STATTM User's Guide, Release 6.03 Edition. SAS Institute Inc., Cary, NC.

Winer, J.A. \& Bahn, S. (1967) Loss of teeth with antidepressant drug therapy. Arch. Gen. Psychiatry., 16, 239-240. 